\title{
On naturalistic pharmacogenetic studies: reply from the authors
}

A letter in response to: Ruaño G, Szarek BL, Villagra D et al. Length of psychiatric hospitalization is correlated with CYP2D6 functional status in inpatients with major depressive disorder. Biomarkers Med. 7(3), 429-439 (2013).

We appreciate the insightful and constructive comments regarding our published work [1] linking length of psychiatric hospitalization for treatment of major depressive disorder (MDD) to CYP2D6gene functional status, as forwarded in letters to the editor by LLerena $e t$ al. [2] and Duconge et al. [3]. Our research approach compared the patient outcome of length of hospitalization in patients with absent or low functional status with patients with normal or high functional status, as determined by CYP2D 6 genotype status. We discovered that subnormal metabolizers had a longer hospitalization by 2 days.

The contributors of the letters praised our study for having carried the research to examine possible relationships between CYP2D6genotype test result and patient outcomes in a naturalistic setting. Such an approach necessarily decreases experimental control and runs the risk of having limitations. The contributors therefore rightfully identified study design issues and analytical considerations that warrant caution in generalizing the results.

Duconge et al. raised concern that the quasiexperimental design employed in our observational study and the ethnic diversity of the patients limits the generalizability of the results. Indeed, our design allowed patients from distinctly different ethnogeographic backgrounds or from a mixed ethnic background, and with or without a variety of other psychiatric diagnoses in addition to the diagnosis of MDD, to enter the study. Such heterogeneity may influence the length of hospitalization outcome. We acknowledge that our naturalistic study approach prevented the control of such factors and that such factors may affect the length of hospitalization. LLerena et al. raised concerns about possible bias in frequencies of CYP2D 6 alleles owing to the selection of patients at a single treatment center and about the confounding effects of comedications and psychopathology, including severity of suicidal tendency. We agree with these authors, and in fact had included these limitations in the 'Perspective on clinical complexities' section in the Discussion section of the paper. In addition, the relationship raised by LLerena et al. between pharmacokinetic pathways and neurotransmitter levels is certainly noteworthy and will require consideration in future research.

Economic pressures to safely reduce length of hospitalization while avoiding readmission within 30 days pertain to all major psychiatric diagnoses including MDD. Our study findings suggest that CYP2D6 genotype-guided prescribing in patients with MDD may have the potential to reduce healthcare costs.

A recently published study from Denmark demonstrated pharmacogenetic-economic relationships in patients with schizophrenia. Herbild et al. tested patients for variants in CYP2D6 and CYP2C19 and randomly assigned them to two groups [4]. One group was treated using pharmacogenetic-guided data on these genes and the other without such information. Pharmacogenetic-guided treatment consisted of informing psychiatrists about the genotype of the patients. Depending on a patient's genotype, the treating psychiatrist was recommended to follow a specific treatment approach outlined in a set of clinical guidelines. Total costs of treatment for 1 year in the PGx group (mean: US\$23,361) were $76 \%$ of those compared with the control group (mean: US $\$ 27,350$; trend; $\mathrm{p}=0.072$ ). The study also found that extreme metabolizers (poor and ultrarapid) prescribed with pharmacogenetic guidance had reduced costs of pharmaceuticals (53\%) compared with nonextreme metabolizers. From our paper and this Danish study, the evidence continues to mount that pharmacogenetics matters both clinically and economically.

In summary, we are grateful for the crossdisciplinary interest evident from these authors

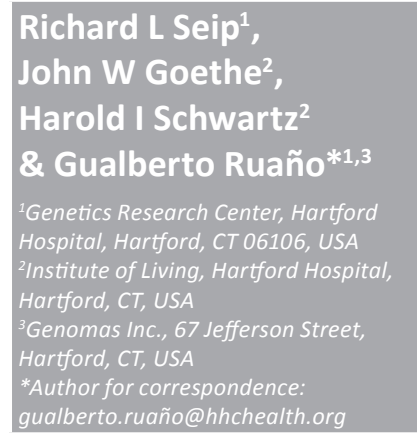

Future
Medicine
part of 
and the scientific community at large, as we seek to advance personalized care for the mentally ill. We look forward to translating pharmacogenetics to the standard of care in psychiatry.

\section{Disclaimer}

This work is the opinion of the authors and does not represent the views of Future Medicine or its employees.

Financial \& competing interests disclosure

Funds for this study were provided by Hartford Hospital Research Program grant \#HH 050921 'The Utility of
Routine Cytochrome P450 Genotyping in the Selection of Type and Dosage of Antidepressant Medication' awarded to JW Goethe as Principal Investigator. This study was also supported by NIH grant 2 R44 MH073291 to G Ruaño. $G$ Ruaño is President and Founder of Genomas Inc. The authors have no other relevant affiliations or financial involvement with any organization or entity with a financial interest in or financial conflict with the subject matter or materials discussed in the manuscript apart from those disclosed.

No writing assistance was utilized in the production of this manuscript.

\section{References}

1 Ruaño G, Szarek BL, Villagra D et al. Length of psychiatric hospitalization is correlated with $C Y P 2 D 6$ functional status in inpatients with major depressive disorder. Biomarkers Med. 7(3), 429-439 (2013). patients' hospitalization period. Biomarkers Med. 7(6), 915-916 (2013).

3 Duconge J, Cadilla CL. CYP2D6's functional status associated with the length of hospitalization stay in psychiatric patients: a twist in the tale or evidence that matters? Biomarkers Med. 7(6), 913-914 (2013).
4 Herbild L, Andersen SE, Werge T, Rasmussen HB, Jurgens G. Does pharmacogenetic testing for CYP4502D6 and 2C19 among patients with diagnoses within the schizophrenic spectrum reduce treatment costs? Basic Clin. Pharmacol. Toxicol. 113(4), 266-272 (2013). 